\title{
Non-anatomical intestinal transplantation
}

\author{
R. Charco, C. Malagelada ${ }^{1}$, L. Llopart ${ }^{2}$, J. Bueno, I. Bilbao, M. Caralt, R. Vilallonga ${ }^{3}$ J. Gavaldá4,

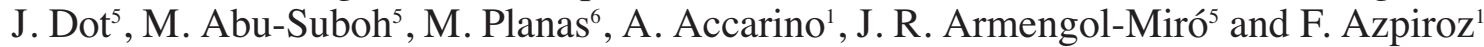

Services of Hepatobiliopancratic and Transplantation Surgery, ${ }^{1}$ Digestive Diseases, ${ }^{2}$ Intensive Care, ${ }^{3}$ Infectious Diseases, ${ }^{4}$ General Surgery, ${ }^{5}$ Digestive Endoscopy and ${ }^{6}$ Unit of Nutritional Support. University Hospital Vall d'Hebron.

Universidad Autónoma de Barcelona. Barcelona, Spain

\begin{abstract}
Introduction: intestinal transplantation is the only long-range treatment option for patients with intestinal failure. We report an exceptional case of isolated intestinal transplantation with the implant in a non-anatomical position.

Clinical case: the patient was a thirty-eight-year-old man (60 $\mathrm{kg}$ weight, $180 \mathrm{~cm}$ height, 18.3 body mass index) with intestinal failure and home parenteral nutrition due to a short-bowel syndrome for which intestinal transplantation was indicated. The patient had a vascular malformation with the cava vein located left to the aorta, and the intestine was implanted with a $180^{\circ}$ rotation around the mesenteric axis, so that the implant's superior mesenteric artery and vein matched the recipient's cava and aorta. Postoperative follow-up was excellent and the patient was discharged after six weeks with a $10-\mathrm{kg}$ gain in body weight.

Discussion: this non-anatomical intestinal implantation of the small bowel, previously unreported, offers technical advantages over other options. Adequate intestinal function represents a unique model to prove the viability of intestinal implants in a nonanatomical position.
\end{abstract}

Key words: Intestinal transplantation. Vascular transposition. Thymoglobulin.

Charco R, Malagelada C, Llopart L, Bueno J, Bilbao I, Caralt $M$, Vilallonga R, Gavaldá J, Dot J, Abu-Suboh M, Planas M, Accarino A, Armengol-Miró JR, Azpiroz F. Non-anatomical intestinal transplantation. Rev Enferm Dig 2009; 101: 139-143.

\section{INTRODUCTION}

Intestinal transplantation is the only long-range treatment option for patients with intestinal failure in whom parenteral nutrition fails due to complication or is insuffi-

Recibido: 18-11-08.

Aceptado: 19-11-08.

Correspondencia: Ramón Charco. Servicio de Cirugía HBP y Trasplantes. Hospital Vall d'Hebron. Paseo Vall d'Hebron, 119-129. 08035 Barcelona, Spain.e-mail: rcharco@vhebron.net cient to cover nutritional requirements $(1,2)$. Because of the large amount of lymphocytes in intestinal immune systems, rejection rate is high and results in intestinal damage, bacterial translocation, and sepsis (3), which can lead to implant failure and death. The high immunogenicity of the small bowel requires tight compatibility between recipient and implant. Furthermore, when the indication for transplantation is short-bowel syndrome, the donor should be smaller than the recipient to prevent spatial compromise. The problem of implant viability, characteristic of intestinal transplantation, has impaired its development as compared with other transplants. We report a case in which, for the first time, an intestinal implant was rotated $180^{\circ}$ around its mesenteric axis to match a congenital malformation in the recipient. This non-anatomical implantation, with no previous reports in the literature, offers technical advantages over other options, but also has potential problems regarding implant function.

\section{CASE REPORT}

The patient, a 38-year-old male with intestinal failure and parenteral nutrition for 18 months, had undergone surgical treatment for morbid obesity in 2002. After 4 years of favorable outcome and weight reduction, the patient presented with intestinal volvulus that required resection of the whole small bowel and right colon. The GI tract was closed at the level of the descending duodenum without continuity with the transverse colon; gastric draining was allowed via a gastrostomy. Due to the loss of the entire small bowel, the patient required home parenteral nutrition with no potential for intestinal rehabilitation. The patient was referred to our center for assessment regarding intestinal transplantation. An isolated small-bowel transplant was indicated based on patient clinical circumstances and considering that he had experienced recurrent sepsis episodes during the last year. The patient was included in the waiting list with $60 \mathrm{~kg}$ weight, $180 \mathrm{~cm}$ height, and 18.3 body mass index. 
Two months later an intestinal transplant was performed with an isogroup donor. The donor was administered thymoglobulin $2 \mathrm{mg} / \mathrm{kg}$ for optimal lymphocyte depletion in order to reduce rejection or implant-versushost reaction risks. The recipient had transposition of the great vessels with the cava vein located left to the aorta. This problem was approached by a $180^{\circ}$ rotation of the implant around the mesenteric axis, so that the superior mesenteric artery-vein in the implant matched the aorta and cava vein in the recipient, respectively (Fig. 1). The first jejunal loop of the implant was anastomosed laterolaterally to the descending duodenum, and the terminal ileum of the implant latero-terminally to the transverse colon, leaving a venting ileostomy to facilitate access to the intestinal mucosa for serial biopsy follow-up. Due to the implant rotation, the ileostomy was located on the right, rather than the left, side of the abdomen. Feeding jejunostomy and cholecystectomy - bile sludge occurs very frequently in this type of patients - were also performed.

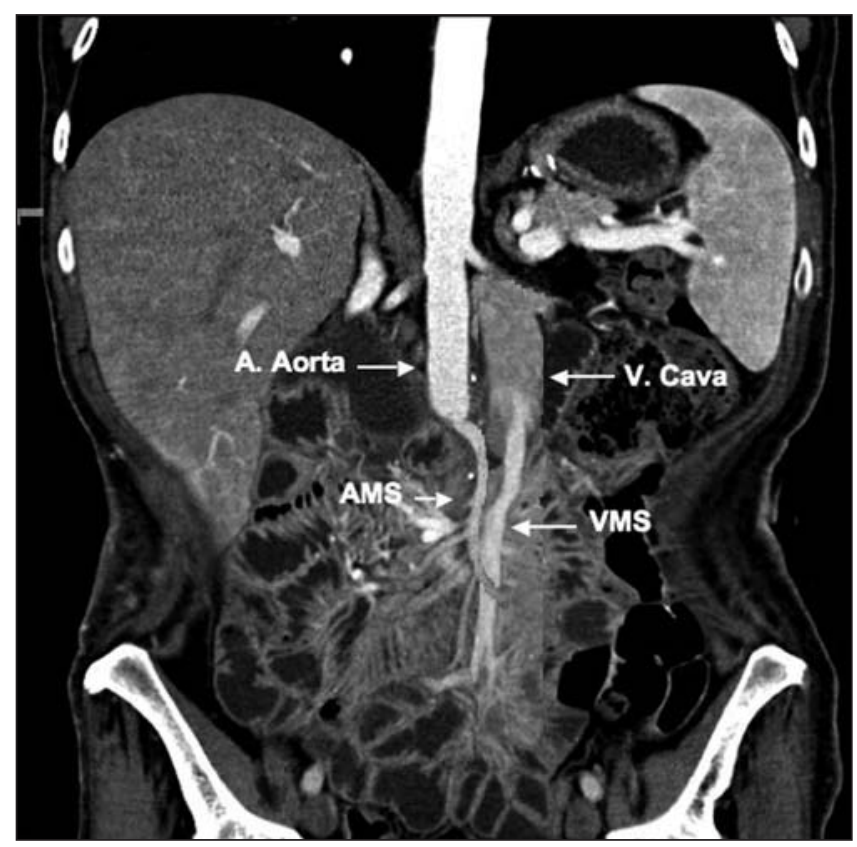

Fig. 1. Helical CT scan. Vascular image of the implant in a non-anatomical position. Note the aorta on the right side of the cava vein.

TAC helicoidal. Imagen vascular del implante intestinal en posición no anatómica. Arteria aorta a la derecha de la vena cava.

The patient received induction treatment with thymoglobulin $1.5 \mathrm{mg} / \mathrm{kg}$ and antibiotic prophylaxis with vancomycin, aztreonam, and liposomal amphotericin B. During the immediate postoperative period the patient received triple immunosuppresor therapy: thymoglobulin $1.5 \mathrm{mg} / \mathrm{kg} /$ day (for 5 days); tacrolimus $0.15 \mathrm{mg} / \mathrm{kg} / \mathrm{day}$ (in two doses) via the nasogastric tube, with doses titrated to reach blood levels of $15-20 \mathrm{ng} / \mathrm{dl}$; and prednisolone
$200 \mathrm{mg} /$ day in a protocol descending down to $20 \mathrm{mg}$ on the $6^{\text {th }}$ day. Since on the $3^{\text {rd }}$ postoperative day tacrolimus blood levels were not appropriate, the drug was administered intravenously for four days for correction before returning to the enteral route. PGE1 (Alprostadil ${ }^{\circledR}$ ) was also administered to improve reperfusion injury (4).

Seventy-two hours after transplantation the patient presented with upper gastrointestinal hemorrhage due to a cardial ulcer that was treated with an endoscopic clip. Ten days after transplantation the patient was discharged from the intensive care unit to the ward. Intestinal biopsies were performed per protocol via the ileostomy twice a week during the first month, and once a week during the second month. Only one biopsy showed indeterminate rejection, and another biopsy revealed mild rejection, which was treated with three boluses of methylprednisolone $500 \mathrm{mg}$.

From a nutritional point of view, on the $7^{\text {th }}$ day posttransplantation water tolerance was tested, followed by enteral nutrition via the feeding jejunostomy. The third week postoperatively the patient started oral feeding, progressively up to 2,100 kcal per day, which allowed patenteral nutrition discontinuation. The patient gained $10 \mathrm{~kg}$ of body weight over 6 weeks, and was discharged at the $7^{\text {th }}$ week without requiring parenteral nutrition. To this day his postoperative course has been excellent, with weight gain supporting the viabilitity and proper function of the intestinal implant.

\section{DISCUSSION}

Intestinal transplantation has a low prevalence due to implant viability issues and a low number of candidates (1). In 20 years 2,000 procedures have been performed worldwide, and this represents the only transplant procedure that is more frequent in the pediatric population (1). Intestinal transplantation is indicated in case of parenteral nutrition complications $(2,3)$ : severe hepatic dysfunction, central venous thrombosis, or repeat sepsis, as in our patient.

In our case, only the small bowel was transplanted, but in cases of gut motor disorders small-bowel transplantation is associated to stomach and/or colon transplants. In case of irreversible hepatic damage from parenteral nutrition (more frequent in children), the liver should also be transplanted $(2,3)$. The introduction of tacrolimus as an immunosuppressor in the nineties, and the systematic use of induction protocols (anti-CD25, thymoglobulin, Campath $1-\mathrm{H}$ ) have reduced the incidence of rejection, and increased survival $(3,5,6)$. At present, more than $80 \%$ of patients can perform normal daily activities and tolerate full oral feeding $(1,2,3,6)$.

Our patient exhibited a vascular malformation that posed an exceptional technical problem. The location of the implant in an anatomical position would have re- 
quired two vascular implants (one venous and other arterial) to communicate the superior mesenteric artery and vein with the aorta and the cava vein, respectively, with the resulting risk of vascular thrombosis. As an alternative option, the implant was rotated around its mesenteric axis. The abdominal cavity behaves as a reservoir containing a viscous fluid with a density similar to water. Flotation forces counteract gravitational forces, and under these conditions the implant, with the same density as the surrounding viscous fluid, is free of postural influences. Adequate, long-term intestinal function indicates the validity of this hypothesis. This case is a unique model to prove the viability of a bowel implant in a nonanatomical position.

\section{REFERENCES}

1. Intestinal Transplant Registry data. Available at: http://www.intestinaltransplant.org

2. Pascher A, Kohler S, Nehaus P, Pratschke J. Present status and future perspectives of intestinal transplantation. Transplant Int 2008; 21: 401-14.

3. Fryer JP. The current status of intestinal transplantation. Curr Opin Organ Transplant 2008; 13: 266-72.

4. Majno PE, Licker M, Kadry Z, Buhler L, Morel DR, Morel P. Effect of dopamine, norepinephrine, and prostaglandin-E1 on graft perfusion in small bowel transplantationin pigs. Trasplant Proc 1996; 28: 2549-50.

5. Fishbein TM, Florman S, Gondolesi G, Schiano T, LeLeiko N, Tschernia $\mathrm{A}$, et al. Intestinal transplantation before and after introduction of sirolimus. Transplantation 2002; 27: 1538-42.

6. Abu-Elmagd K, Reyes J, Bond G, Mazariegos G, Wu T, Murase N, et al. Clinical intestinal transplantation: a decade of experience at single center. Ann Surg 2001; 234: 404-16. 